\title{
Registration based filtering: an acceptable tool for noise reduction in left ventricular dynamic rotational angiography images?
}

\author{
Jean-Yves Wielandts*a,b, Stijn De Buck ${ }^{\mathrm{a}, \mathrm{b}}$, Joris Ector ${ }^{\mathrm{a}}$, Dieter Nuyens ${ }^{\mathrm{a}}$, Frederik Maes ${ }^{\mathrm{b}, \mathrm{c}, \mathrm{d}}$, Hein \\ Heidbuchel $^{\mathrm{a}}$ \\ ${ }^{a}$ Department of Cardiovascular Sciences, KU Leuven; ${ }^{b}$ Medical Imaging Research Center, KU \\ Leuven \& UZ Leuven; ${ }^{\mathrm{C}}$ Department of Electrical Engineering, ESAT/PSI, Medical Image \\ Computing, KU Leuven; ${ }^{\text {i }}$ Minds-Future Health Department, KU Leuven; Leuven, Belgium
}

\begin{abstract}
VT ablations could benefit from Dynamic 3D (4D) left ventricle (LV) visualization as road-map for anatomy-guided procedures. We developed a registration-based method that combines information of several cardiac phases to filter out noise and artifacts in low-dose 3D Rotational Angiography (3DRA) images. This also enables generation of accurate multi-phase surface models by semi-automatic segmentation (SAS).

The method uses B-spline non-rigid inter-phase registration (IPR) and subsequent averaging of the registered 3DRA images of 4 cardiac phases, acquired with a slow atrial pacing protocol, and was validated on data from 5 porcine experiments. IPR parameter settings were optimized against manual delineations of the LVs using a composed similarity score (Q), dependent on DICE-coefficient, RMSDistance, Hausdorff (HD) and the percentage of inter-surface distances $\leq 3 \mathrm{~mm}$ and $\leq 4 \mathrm{~mm}$. The latter are clinically acceptable error cut-off values.

Validation was performed after SAS for varying voxel intensity thresholds (ISO), by comparison between models with and without prior use of IPR. Distances to the manual delineations at optimal ISO were reduced to $\leq 3 \mathrm{~mm}$ for $95.6 \pm 2.7 \%$ and to $\leq 4 \mathrm{~mm}$ for $97.1 \pm 2.0 \%$ of model surfaces. Improved quality was proven by significant mean Qincrease irrespective of ISO $(7.6 \%$ at optimal ISO $(95 \% \mathrm{CI} 4.6-10.5, \mathrm{p}<0.0001))$. Quality improvement was more important at suboptimal ISO values. Significant $(\mathrm{p}<0.0001)$ differences were also noted in HD $(-20.5 \%$;95\%CI $-12.1 \%$-$29.0 \%)$, RMSD (-28.3\%;95\%CI -21.7\%--35.0\%) and DICE (1.7\%;95\%CI 0.9\%-2.6\%). Generating 4D LV models proved feasible, with sufficient accuracy for clinical applications, opening the perspective of more accurate overlay and guidance during ablation in locations with high degrees of movement.
\end{abstract}

Keywords: Rotational Angiography, cardiac ablation, prospective gating, C-arm imaging, cone-beam, 4D imaging, interventional imaging, semi-automatic segmentation, left ventricle.

\section{INTRODUCTION}

Three Dimensional Rotational Angiography (3DRA) has become an established modality for 3D imaging of the heart, particularly for use during catheter ablation procedures, due mainly to its high anatomic detail notwithstanding a relatively low radiation burden and its advantages in terms of logistics and patient comfort. ${ }^{1}$ High resolution X-ray images, per-procedurally acquired through $\mathrm{C}$-arm rotation around the patient, can be reconstructed and segmented into a 3D model. Subsequently, this model can be used in a separate electro-anatomical mapping system or in overlay with live fluoroscopy during the procedure to offer on line visual feedback of catheter positions supported by highly detailed 3D anatomy. ${ }^{2,3}$

Dynamic 3D (4D) imaging can, however, enable more accurate anatomical location of catheters throughout the heart cycle, avoiding positional mismatch caused by the overlay with a static 3D model, while retaining high anatomical detail. This advantage can be especially important during ablation in locations with a high degree of movement. We recently developed a new prospective imaging technique that is capable of imaging the dynamics of the cardiac cavities during the intervention with a relatively low radiation dose. Prospective 4DRA can now be established by applying slow

Medical Imaging 2014: Image-Guided Procedures, Robotic Interventions, and Modeling,

edited by Ziv R. Yaniv, David R. Holmes III, Proc. of SPIE Vol. 9036, 903628

(C) 2014 SPIE · CCC code: $1605-7422 / 14 / \$ 18 \cdot$ doi: 10.1117/12.2043480 
atrial pacing to obtain a stable heart rhythm and a single 200 degree $\mathrm{C}$-arm rotation with imaging at a regular imaging interval. First results have been published indicating acceptable surface distance errors when comparing manual delineations of the left ventricle (LV) in the 4DRA images with the according MR images. ${ }^{4}$

We explored the potential of B-spline non-rigid registration for combining information of multiple cardiac phases (inter-phase registration - IPR) to filter out noise and artifacts inherent to low-dose 3DRA images. This would in turn facilitate the generation of accurate multi-phase surface models by means of semi-automatic segmentation (SAS). This work focusses on the left ventricle (LV), as it is the cardiac chamber with the most prominent motion throughout the cardiac cycle, while accurate LV ablations often constitute life-saving procedures.

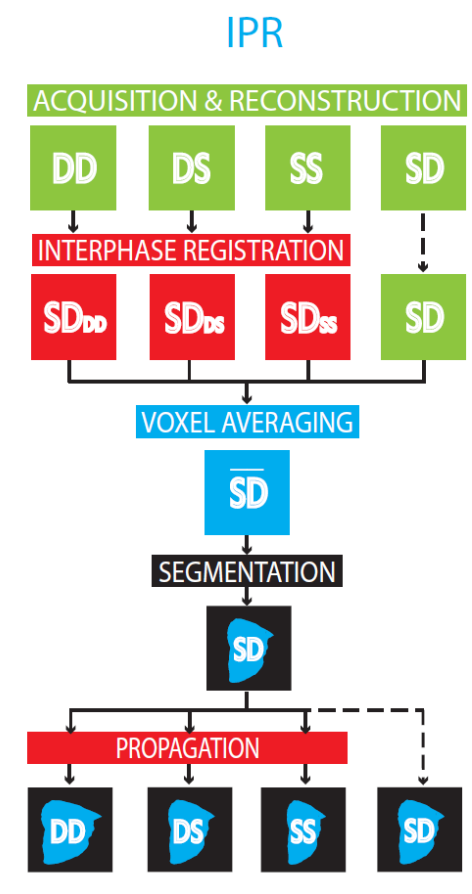

(A)

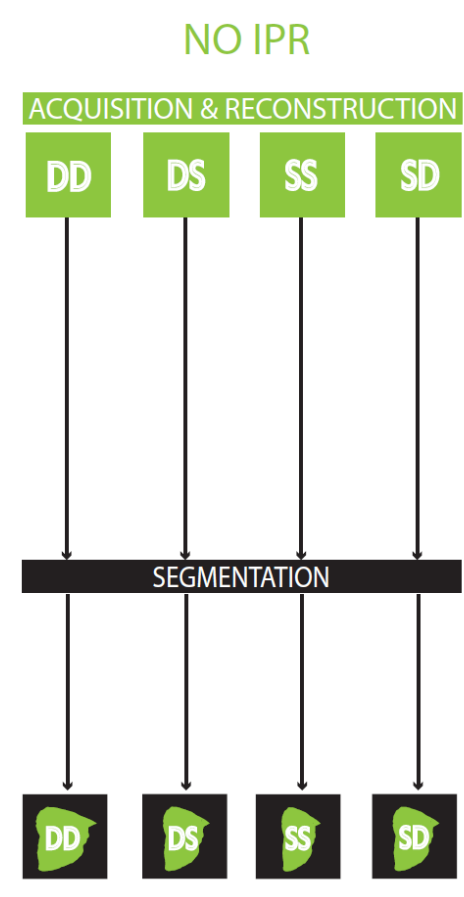

(B)

Figure 1. (A) Interphase registration (IPR) based filtering method: the reconstructed images of 3 phases are registered to the anchor phase (SD). Subsequent image averaging is performed to increase SNR prior to semi-automatic segmentation. The surface models of the 3 other phases are obtained by propagation using the transformation defined in the IPR step. (B) Direct semi-automatic segmentation of the reconstructed images for the 4 selected phases separately.

\section{METHODOLOGY}

\subsection{Method Description}

The proposed method for noise reduction is constituted of a number of steps as schematically visualized in Figure 1A. Image acquisition was performed using a Siemens Artis System (Siemens Medical, Forcheim, Germany) using our recently developed single 200 degree C-arm rotation 3DRA image acquisition protocol. ${ }^{4}$ Five pigs were anaesthetized and paced at $105 \mathrm{bpm}$ (i.e. a pacing interval of $574 \mathrm{~ms}$ ) through a pacing catheter positioned in the right atrium. Between 90 and $150 \mathrm{ml}$ of undiluted iodine contrast agent (Iomeron 300, Bracco, Milano, Italy) were administered through the femoral vein. A 3D+t stack of 15 distinct cardiac phases was reconstructed on a dedicated Siemens workstation by means of an algorithm similar to the one presented by Feldkamp et al., ${ }^{5}$ using 25 or 26 images per phase. Acquiring 15 phases in a clinical setting would however imply an unacceptably high (estimated) radiation dose of $\geq 10 \mathrm{mSv}$, even with 
a low dose image acquisition protocol. ${ }^{1,6,7}$ We therefore performed this work on 4 phases only since they may suffice in covering the mechanical variation of the heart's shape throughout its cycle. The 4 selected phases were the diastolic (DD), the systolic (SS), and two interposed phases selected halfway ventricular contraction (DS) and relaxation (SD) respectively.

In the first step, a registration is computed between the reconstructed image of a selected phase (anchor phase) and the remaining three phases by means of B-spline non-rigid registration. In a second step the anchor phase image and the 3 co-registered images are averaged voxel-by-voxel to create a single phase image with reduced noise and artifacts. Next, semi-automated segmentation with an in-house developed software (EPSegmenter, previously reported in De Buck S. et l. $^{4}$ ), was done to extract the target volume and generate a 3D surface model of the single phase image. This model can then be used in the final step to generate the segmentations of the remaining 3 phases, using the known transformations defined in the registration step. The practical implementation of the inter-phase B-spline non-rigid registration was done using the Elastix toolbox. ${ }^{8}$ Advanced Mattes Mutual Information was used as metric and $3^{\text {rd }}$ order B-spline as interpolator, while the number of iterations, control point spacing, multi-resolution strategy and number of spatial samples for the adaptive stochastic gradient descent optimizer were considered tunable parameters.

\subsection{Method Optimization}

A ground truth set of segmentations was first created by manual delineation of the LVs in the 5 experiments for all 4 phases on the reconstructed images. The considered region of interest (ROI) spans the LV from apex to basis, proximal of LV in- and outflow tract. This delineation was done in a per slice manner, after resampling the image volume along $1 \mathrm{~mm}$ thick slices orthogonal to the $\mathrm{LV}$ long axis with a voxel resolution of $1 \mathrm{~mm}^{3}$. Different combinations ( $\mathrm{n}=78$ ) of the tunable parameters were used to perform phase-to-phase registration in all phase-pairs $(n=12)$. In order to evaluate the parameter sets, the spatial transformations defined in this registration step were also applied to the manual delineations of every phase for comparison to the ground truth delineation of every other phase.

Comparison was done using 5 different similarity measures: Sørensen-Dice coefficient (DICE), Root Mean Square of inter-surface distances (RMSD), Hausdorff Distance (HD) and the percentage of inter-surface distances $\leq 3 \mathrm{~mm}$ and $\leq 4 \mathrm{~mm}(\mathrm{~d} 3 \mathrm{~mm}$ and $\mathrm{d} 4 \mathrm{~mm}$ respectively). The latter two measures were selected because they represent the amount of surface points within the range of typical LV ablation lesion width and depth, which easily reach $4 \mathrm{~mm}$ and $3 \mathrm{~mm}$ respectively. ${ }^{9}$ In order to obtain an overall quality measure incorporating these 5 measures, principal component analysis was performed on the measures computed for the data resulting from the application of every parameter set in all 4 phases for the 5 experiments. A composite score $\mathrm{Q}$ was then defined along the first eigenvector. The reference phase that on average resulted in the highest $\mathrm{Q}$ values was selected as anchor phase for all experiments. The same criterion was used to select the optimal set of IPR parameters for each of the remaining 3 phases separately. An IPR calculation time of 2 minutes on a desktop PC (Dual Intel Xeon 6-core 2.30Ghz processors at 64-bit, 32Gb RAM, Dell, Texas, USA) was selected as reasonable threshold for on line clinical usability.

\subsection{Method Validation}

In order to assess the impact of our IPR-based filtering method for noise and artifact reduction, a 4-phase surface model sequence was generated in two distinct ways (see Figure 1): (A) SAS was performed on the anchor phase alone and the segmentations of the three remaining phases were obtained as described above, resulting in a sequence of 4 phase segmentations (IPR-sequence), (B) SAS was performed directly on the reconstructed images in all 4 phases separately (no-IPR sequence). The solutions obtained through (A) and (B) were then compared quantitatively using the similarity measures vs. the ground truth delineations used in the optimization step. The SAS was done for the range of voxel intensity thresholds (isosurface value, ISO) generating 3D models deemed clinically usable by a cardiologist for each experiment separately. To facilitate the analysis of segmentation accuracy between different experiments, the ISO values for each experiment are expressed as offsets $\triangle \mathrm{ISO}$ relative to the optimal ISO value without the use of IPR, i.e. $\Delta \mathrm{ISO}=0$ corresponds to the ISO value for which SAS of the original phase images resulted on average in the highest composite score Q. 

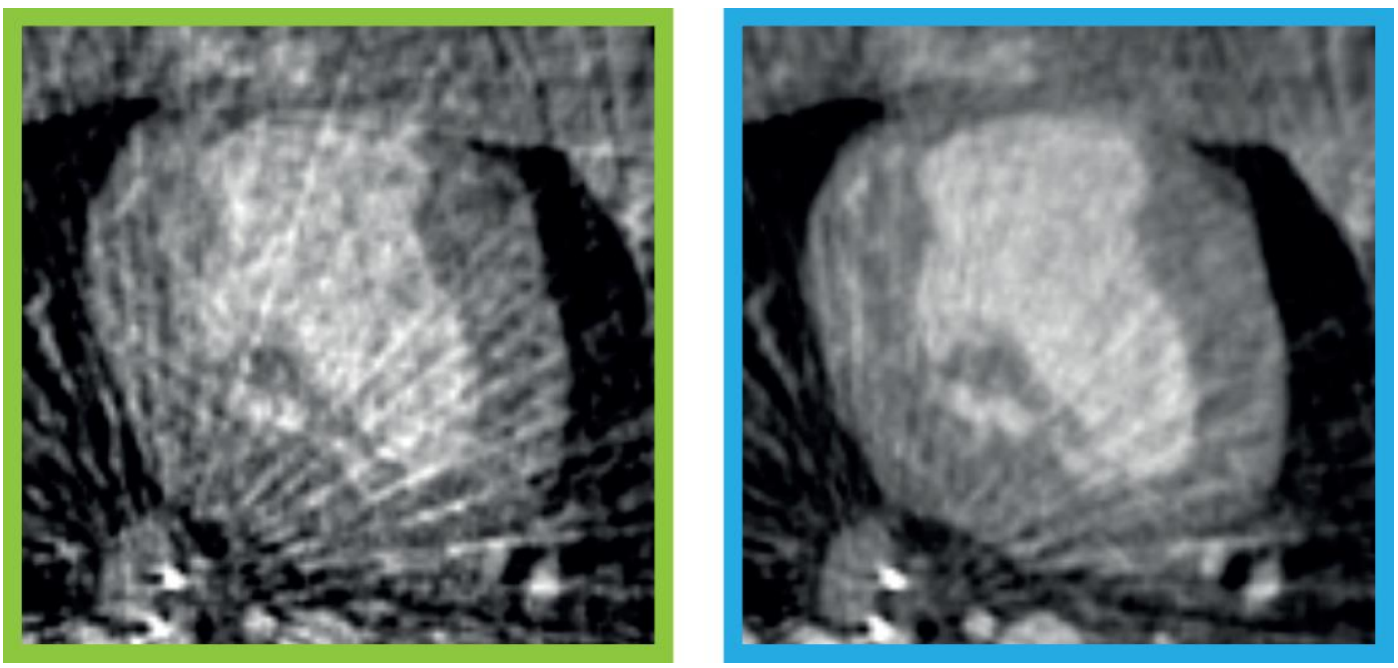

Figure 2. Axial slice through the iodine contrast agent-filled left ventricle in a reconstructed image before (left) and after (right) application of the IPR-based filtering method.

\section{RESULTS}

\subsection{Method Optimization}

The optimal anchor phase and the optimal set of IPR parameters for all other phases were determined by assessing the similarity between the ground truth LV delineations after registration, using the composite score Q. Both interposed phases SD and DS resulted in the highest Q values. However, SD was selected as best anchor phase as the optimal set of IPR parameters was the same for all 3 other phases in case of SD, but not for DS. A single axial slice through the LV before and after IPR-based filtering is shown in Figure 2.

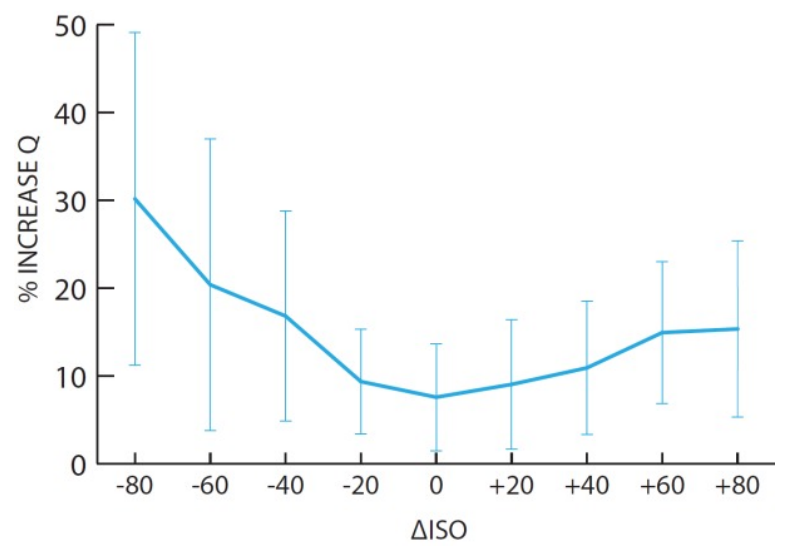

Figure 3. Fractional increase in composite score Q, averaged over all experiments (mean \pm sd), for varying offset ISO value (expressed relative to the optimal no-IPR ISO value). Improved quality in the IPR-sequence compared to the no-IPR sequence increases with $\triangle \mathrm{ISO}$. This indicates an additional robustness for SAS when applying the IPR-based filtering method. 


\subsection{Post-Segmentation Quantitative Validation}

Improved quality in the IPR-sequence was proven by significant relative increase of $\mathrm{Q}$ value compared to the no-IPR sequence and irrespective of voxel intensity thresholds with a mean fractional increase at $\Delta \mathrm{ISO}=0$ of $7.6 \%$ (95\% CI 4.6$10.5 \%, \mathrm{p}<0.0001)$. Quality improvement was more important for $\Delta \mathrm{ISO} \neq 0$ (Figure 3). Significant $(\mathrm{p}<0.0001)$ relative improvement was also noted in all 5 considered similarity measures: HD $(-20.5 \%$ at $\Delta \mathrm{ISO}=0 ; 95 \% \mathrm{CI}-12.1--29.0 \%)$, RMSD $(-28.3 \%$ at $\triangle \mathrm{ISO}=0 ; 95 \% \mathrm{CI}-21.7--35.0 \%)$, DICE $(1.7 \%$ at $\triangle \mathrm{ISO}=0 ; 95 \% \mathrm{CI} 0.9-2.6 \%), \mathrm{d} 3 \mathrm{~mm}(7.8 \%$ at $\Delta \mathrm{ISO}=0$; $95 \% \mathrm{CI} 4.6-10.9 \%)$ and $\mathrm{d} 4 \mathrm{~mm}(6.0 \%$ at $\Delta \mathrm{ISO}=0 ; 95 \% \mathrm{CI} 3.9-8.2 \%)$.

The clinical relevance is shown by the inter-model distances between the IPR sequence and the ground truth set of manual segmentations at $\mathrm{ISO}=0$ being reduced to $\leq 3 \mathrm{~mm}$ for $95.6 \pm 2.7 \%$ and to $\leq 4 \mathrm{~mm}$ for $97.1 \pm 2.0 \%$ of model surfaces with a RMS segmentation error over the whole surface of $1.5 \pm 0.3 \mathrm{~mm}$ at $\Delta \mathrm{ISO}=0$ (see Figure 4 ). The LV models of the 4 phases as obtained without and with the use of IPR are shown for one experiment in Figure 5.
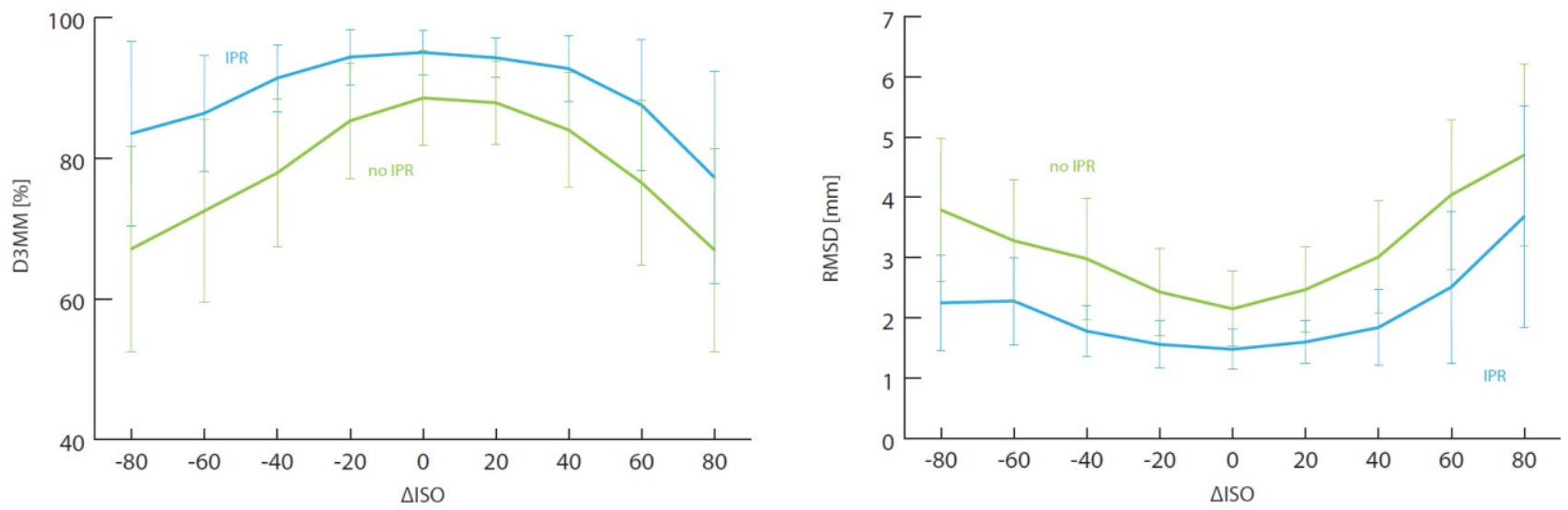

Figure 4. Absolute improvement in similarity (d3mm left, RMSD right) between segmented models and the corresponding manual LV delineation ("ground truth") when applying the IPR-based filtering method. Results are averaged over all experiments (mean \pm sd) and shown for varying offset ISO value.

\section{DISCUSSION}

\subsection{Image Quality}

We present a filtering framework for post-reconstruction noise and artifact reduction in 4DRA images based solely on image registration. Applying this IPR method to pre-process the very noisy reconstructed phase images before semiautomatic segmentation significantly increases their SNR. This results in more accurate models, with a distance to the ground truth delineations of less than $3 \mathrm{~mm}$ for more than $95 \%$ of the model surface and less than $4 \mathrm{~mm}$ for more than $97 \%$, while also mean and maximum error decrease significantly $(-28 \%$ and $-21 \%$ on average respectively). The remaining fraction of inter-surface distances that are $\geq 3 \mathrm{~mm}$ is attributable to residual noise, in particular due to metal streak artifacts not being filtered sufficiently. However, recent advances in iterative reconstruction methods show the possibility of further metal artifact correction, ${ }^{10,11}$ while motion compensation incorporated at the reconstruction stage also shows promising results. ${ }^{12}$

The noise reduction using IPR and voxel averaging leads to a better differentiation between high and low intensity voxels, facilitating semi-automated segmentation as shown by significantly improved similarity measures when comparing to the manually segmented models. In addition, using IPR results in a broader range of ISO values yielding high similarity scores than when IPR is not applied prior to SAS, adding robustness to the segmentation process. 


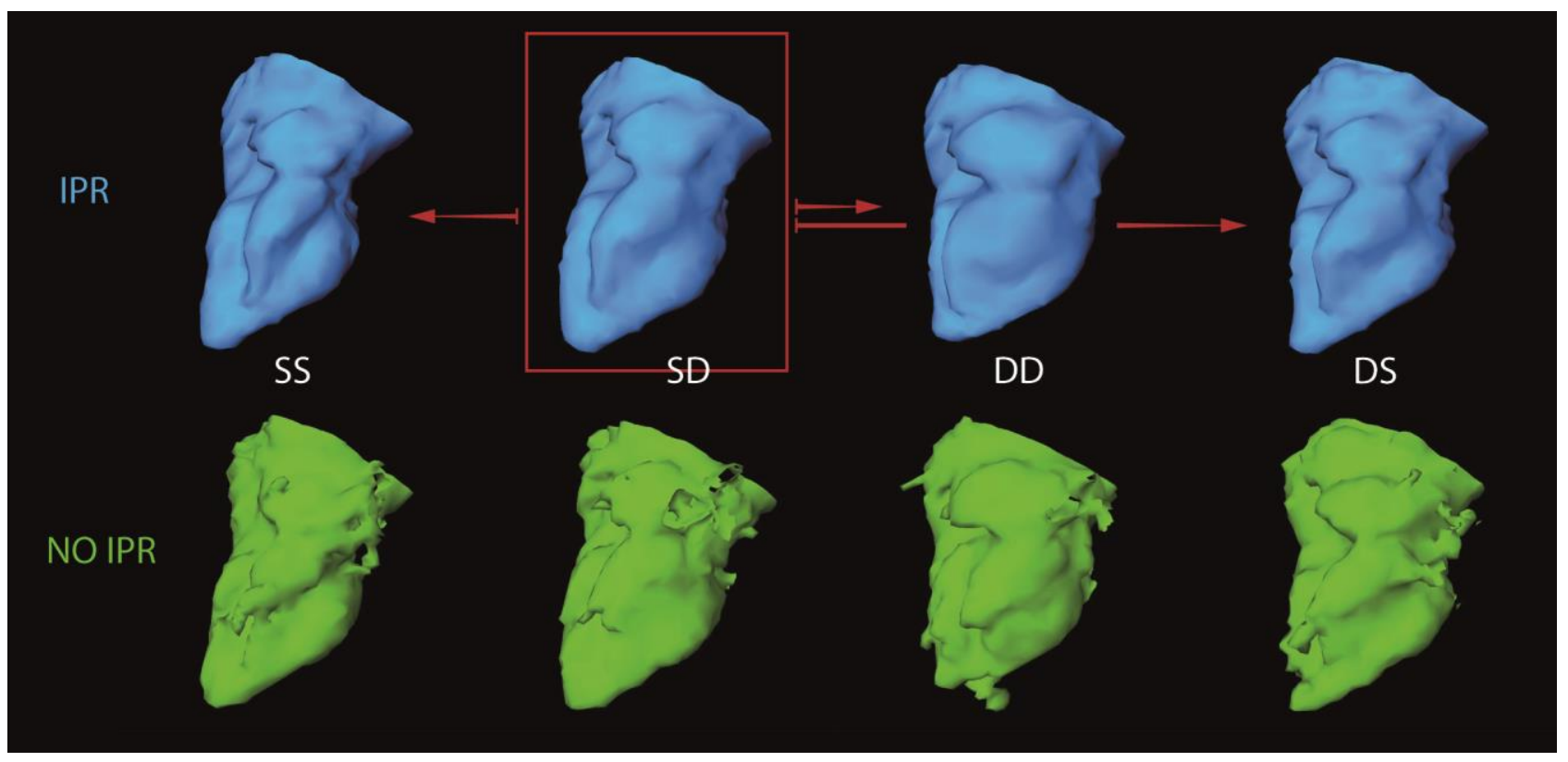

Figure 5. Segmented 4D model sequence of 4 cardiac phases shown for $\Delta \mathrm{ISO}=0$ in one experiment. The IPR sequence is built from the segmentation in the anchor phase (frame) and its propagation to the 3 remaining phases (arrows). The four models in the no-IPR sequence are obtained by direct segmentation of the reconstructed images in all four phases separately.

\subsection{Clinical Implications}

The presented filtering method is validated in a close to realistic clinical workflow with direct quantitative comparison performed at the resulting surface model level. Moreover, the optimization scheme for inter-phase registration parameters is based on generally accepted similarity measures and a newly designed application specific similarity measure.

Static 3D images used in current clinical practice are reconstructed from at least 67 projection images, involving an effective radiation dose of $\pm 2.6 \mathrm{mSv}$ for a clinically useful 3D model, as recently reported in De Buck et al. ${ }^{1}$ As only \pm 100 images would be needed for generating a 4-phase 4D sequence with similar detector entrance dose settings, the expected increase in radiation burden can be estimated not to exceed 50\% compared to static 3D imaging. Slow atrial pacing at a physiologically acceptable heart rate also reduces the risk of inducing unwanted arrhythmias, like ventricular fibrillation, compared to the rapid ventricular pacing approach in static $3 \mathrm{D}$ imaging. ${ }^{2}$

\section{CONCLUSION}

Generating dynamic 3D (4D) models of the left ventricle, by means of 3DRA images, is feasible with clinical accuracy through the use of a new acquisition protocol and subsequent application of B-spline non-rigid inter-phase registration associated with voxel averaging which reduces image noise and artifacts.

We demonstrated a significant impact of the use of IPR on semi-automatic segmentation quality for a 4 phase 4D sequence. Moreover, due to the use of low dose acquisition protocols, radiation dose can be kept at a clinically acceptable level. Also, by using slow atrial pacing the risk of inducing unwanted ventricular tachy-arrhythmias is minimized. These findings open the perspective of realizing a 4D image sequence for more accurate integration with electro-anatomical mapping systems or per-procedural fluoroscopy integration to facilitate catheter ablation in locations with a high degree of motion, like the LV. 


\section{REFERENCES}

[1] De Buck, S., Alzand, B. S., Wielandts, J-Y., Garweg, C., Phlips, T., Ector, J., Nuyens, D., Heidbuchel, H., "Cardiac three-dimensional rotational angiography can be performed with low radiation dose while preserving image quality.," Europace 15(12),1718-1724 (2013).

[2] Ector, J., De Buck, S., Huybrechts, W., Nuyens, D., Dymarkowski, S., Bogaert, J., Maes, F., Heidbuchel, H., "Biplane three-dimensional augmented fluoroscopy as single navigation tool for ablation of atrial fibrillation: accuracy and clinical value.," Heart Rhythm 5:957-964 (2008).

[3] Li, J. H., Haim, M., Movassaghi, B., Mendel, J. B., Chaudhry, G. M., Haffajee, C. I., Orlov, M. V., "Segmentation and registration of three-dimensional rotational angiogram on live fluoroscopy to guide atrial fibrillation ablation: A new online imaging tool.," Heart Rhythm 6:231-237 (2009).

[4] De Buck, S., Dauwe, D., Wielandts, J-Y., Claus, P., Janssens, S., Heidbuchel, H., Nuyens, D., "A new approach for prospectively gated cardiac rotational angiography.," Proc. SPIE 8668 (2013).

[5] Feldkamp, L. A., Davis, L. C., Kress, J. W., "Practical cone-beam algorithm.," J. Opt. Soc. Am. A. 1:612-619 (1984).

[6] Wielandts, J-Y., Smans, K., Ector, J., De Buck, S., Heidbüchel, H., Bosmans, H., "Effective dose analysis of three-dimensional rotational angiography during catheter ablation procedures.," Phys Med Biol. 55:563-579 (2010).

[7] Wielandts, J-Y., De Buck, S., Ector, J., Lagerche, A., Willems, R., Bosmans, H., Heidbuchel, H., "Threedimensional cardiac rotational angiography: effective radiation dose and image quality implications.," Europace 12:194-201 (2010).

[8] Klein, S., Staring, M., Murphy, K., Viergever, M. A., Pluim, J. P., "Elastix: a toolbox for intensity-based medical image registration.," IEEE Trans Med Imaging 29:196-205 (2010).

[9] Wright, M., Harks, E., Deladi, S., Suijver, F., Barley, M., van Dusschoten, A., Fokkenrood, S., Zuo, F., Sacher, F., Hocini, M., Haïssaguerre, M., Jaïs, P., "Real-time lesion assessment using a novel combined ultrasound and radiofrequency ablation catheter.," Heart Rhythm 8:304-312 (2011).

[10] Van Slambrouck, K., Nuyts, J., "Metal artifact reduction in computed tomography using local models in an image block-iterative scheme.," Med Phys. 39:7080-7093 (2012).

[11]Kratz, B., Weyers, I., Buzug, T. M., "A fully 3D approach for metal artifact reduction in computed tomography." Med Phys. 39:7042-7054 (2012).

[12]Müller, K., Rohkohl, C., Lauritsch, G., Schwemmer, C., Heidbuchel, H., De Buck, S., Nuyens, D., Kyriakou, Y., Köhler, C., Hornegger, J., "4-D motion field estimation by combined multiple heart phase registration (CMHPR) for cardiac C-arm data." IEEE, ed. IEEE Nuclear Science Symposium and Medical Imaging Conference Record (NSS/MIC 3707-3712 (2012). 